\title{
Effect of grain packing tightness on strain estimation from the Fry method
}

\author{
Arun Kumar Ojha* and Deepak C Srivastava \\ Department of Earth Sciences, Indian Institute of Technology Roorkee, Roorkee 247 667, India. \\ *Corresponding author.e-mail: ojhaarun323@gmail.com
}

MS received 13 July 2017; revised 21 September 2017; accepted 7 October 2017; published online 25 June 2018

How tightly should a sample be packed for strain estimation by the Fry method? We address this issue using synthetic simulations of 900 images such that each image contains 200 randomly distributed grains, but differs from other images with respect to the packing tightness and the degree of sorting. Each image is coaxially distorted by various known strain ratios and the strain estimates from distorted images are obtained by the Fry method. The statistical errors in the strain estimates are found to grow larger with the decrease in the packing tightness irrespective of the level of distortion. We demonstrate that a progressive decrease in the packing tightness results in an increasingly clustered nature of grain center distribution and hence the larger errors. These results, obtained from the synthetic images, are corroborated by two natural examples of sandstone, one loosely packed and the other tightly packed. Based on the results of tests on synthetic and natural examples, we recommend that the Fry method should be used only on those samples that have $>30 \%$ packing density.

Keywords. Fry method; strain estimation; packing density; sorting; clustering; error estimation.

\section{Introduction}

The Fry method is a graphical technique that uses the relative movement of material points, typically grain centres, for strain estimation in rocks (Fry 1979; Hanna and Fry 1979). The method produces a point distribution, the Fry plot, that contains a characteristic central vacancy, the strain ellipse provided the grains and the matrix are mechanically identical and the distortion is homogeneous (Fry 1979; Lisle 1979; Ramsay and Huber 1983). The Fry method has been extensively used during the last three decades due to its applicability to a wide range of situations and the procedural simplicity (e.g., Lacassin and Van den driessche 1983; Ramsay and Huber 1983; Seno 1992; McNaught 1994; Srivastava 1995; McNaught 2002; Gonzalez-casado et al. 2003; Genier and Epard
2007; Long et al. 2011). Several issues related to the Fry method, such as the degree of anticlustering, the effect of pressure solution, the choice of grain centroid versus grain centre, the use of non-elliptical grains, the degree of sorting, the uncertainty in results and the problem of void fitting have already been addressed in several wellconstrained studies (Crespi 1986; Onasch 1986; Dunne et al. 1990; McNaught 1994, 2002; Ray and Srivastava 2008; Reddy Vinta and Srivastava 2012; Mulchrone 2013).

The problem of subjectivity in the interpretation of the Fry plot, arising due to diffuse boundaries of the central vacancy, was first addressed by Erslev (1988) and Erslev and Ge (1990). Several variants of the Fry method have since been proposed for objective interpretation of the Fry plot. For example, Waldron and Wallace (2007) 


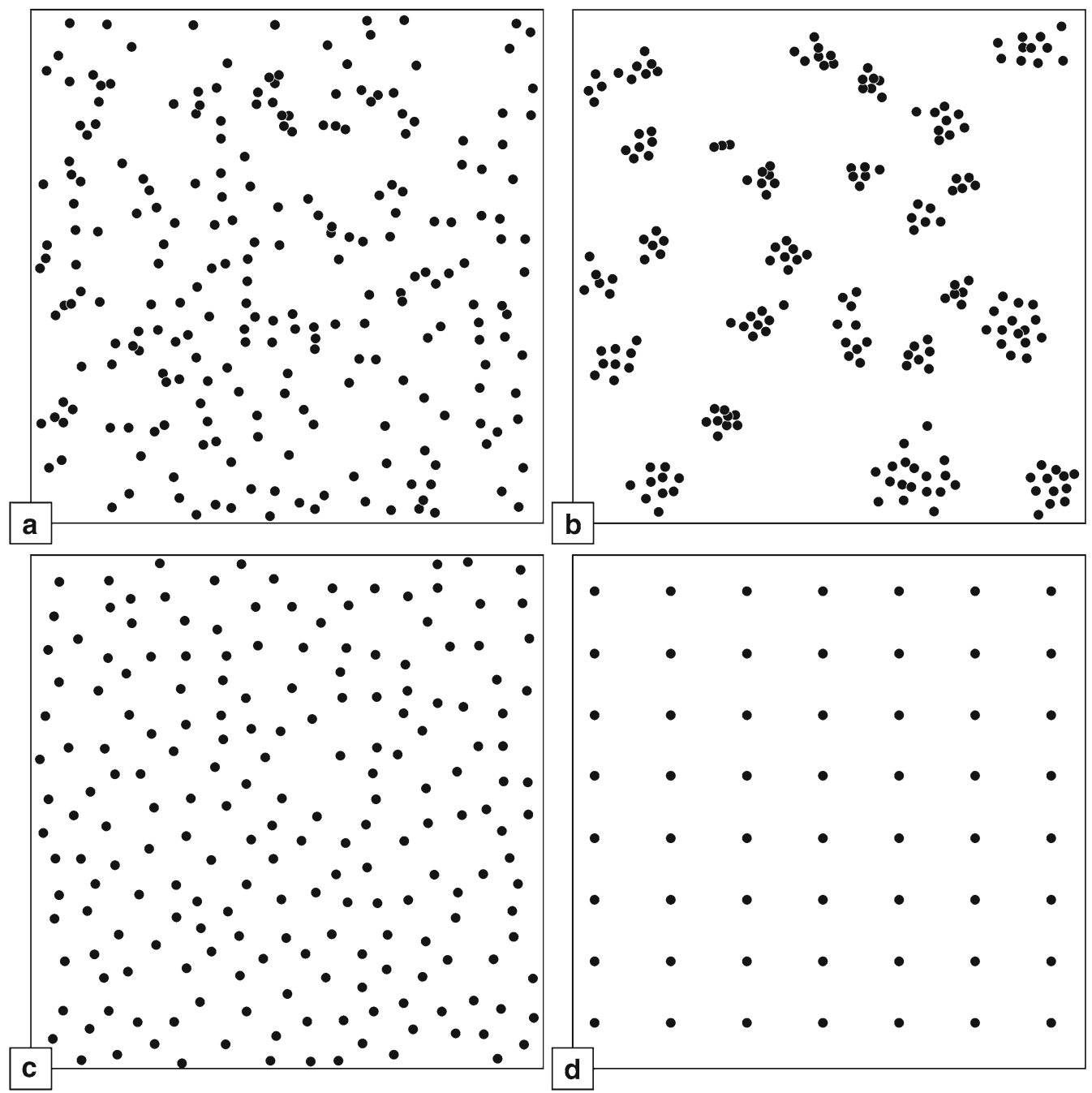

Figure 1. Different types of grain center distribution. (a) Random, (b) clustered, (c) anticlustered isotropic, and (d) anticlustered anisotropic. Modified after Genier and Epard (2007).

proposed a Continuous Function method that identifies the best-fit strain ellipse by the maximum value of an exponential function. In an entirely different approach, Lisle (2010) retro-deforms the Fry plot in successive increments and uses Kuiper's Test and Chi-squared Test for the restoration of the isotropic non-Poisson point distribution. Using a similar approach, Shan and Xiao (2011) give a maximum likelihood method that identifies the truncated Poisson point distribution in the undistorted object set. The image analysis technique of Reddy Vinta and Srivastava (2012) applies a weighted Gaussian blur to each pixel on the Fry plot for extraction of the elliptical central vacancy. Mulchrone $(2003,2013)$ uses the Delaunay nearest neighbour point distribution technique for objective strain estimation from the Fry plot. Kumar et al. (2014) made a comprehensive comparison of these methods and concluded that the Continuous
Function method gives most accurate results. For this reason, we have used the Continuous Function method for strain estimation from Fry plots in all our synthetic and natural examples presented in this study. As the choice of objective variant does not affect the results from the Fry method significantly, the results will hold good irrespective of the object variant chosen.

The nature of grain centre distribution is a critical consideration in the Fry method. The method is most suited to the samples that had an anticlustered grain centre distribution in the undistorted state (Fry 1979). Genier and Epard (2007) test the Fry method on four different types of point distributions:

(i) anti-clustered-isotropic,

(ii) anti-clustered-anisotropic,

(iii) random, and

(iv) clustered (figure 1a-d). 


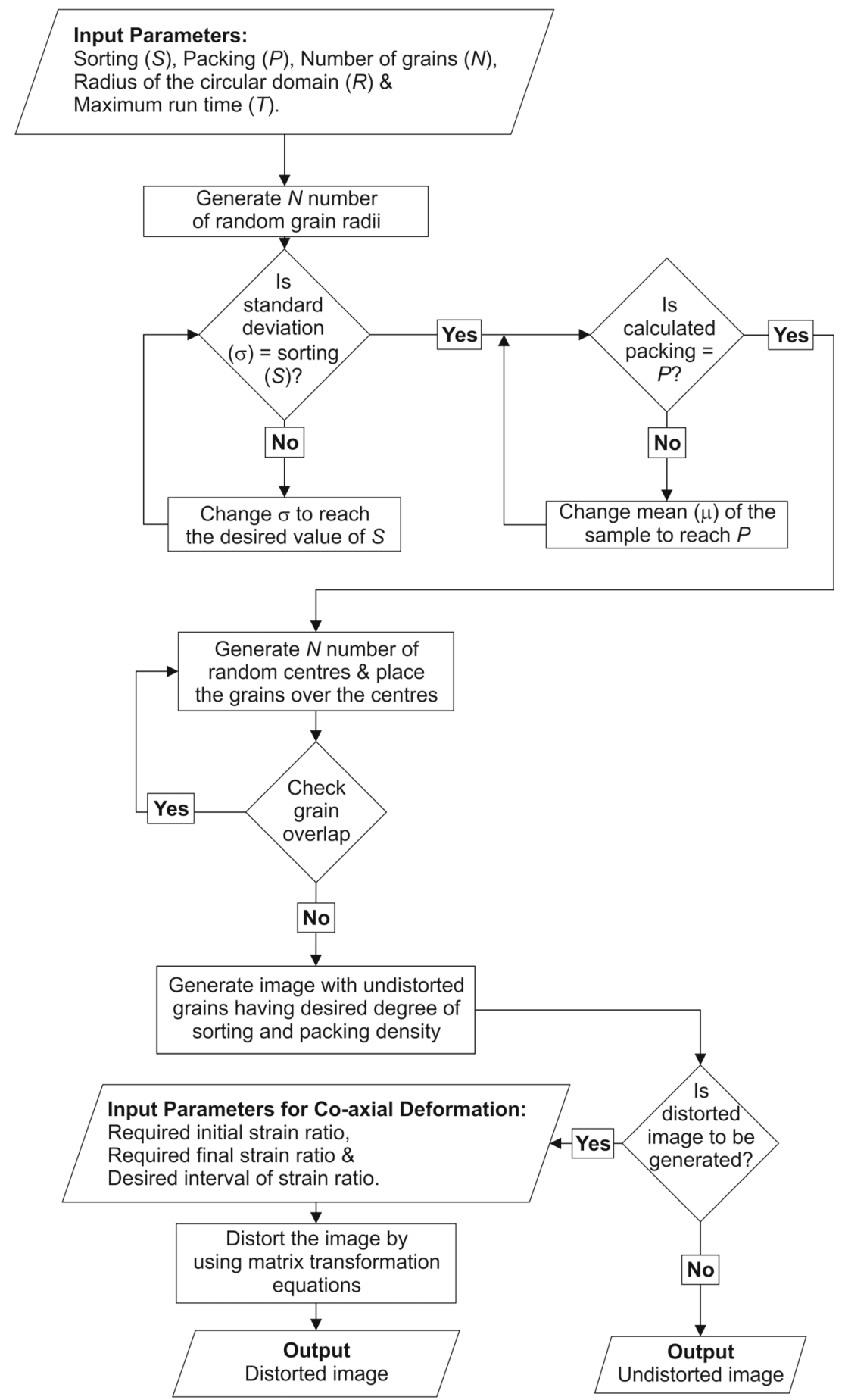

Figure 2. Flowchart of the MATLAB code for simulation of undistorted and distorted images.

Their results confirm that the method gives best results when the grain centre distribution is anticlustered in the undistorted state. Given a distorted sample, it is, however, difficult to predict the nature of the pre-tectonic grain centre distribution without a rigorous analytical treatment (Dacey 1964; Genier and Epard 2007; Lisle 2010). As an alternative, the sedimentological degree of sorting is routinely considered as a proxy for the degree of anti-clustering in the undistorted grain centre distribution (Crespi 1986; McNaught 2002; Reddy Vinta and Srivastava 2012; Mulchrone 2013; Kumar et al. 2014). In general, the well sorted samples are considered suitable for application of the Fry method.

The tightness of grain packing is another important parameter in the selection of a sample for strain estimation by the Fry method (Fry 1979; 

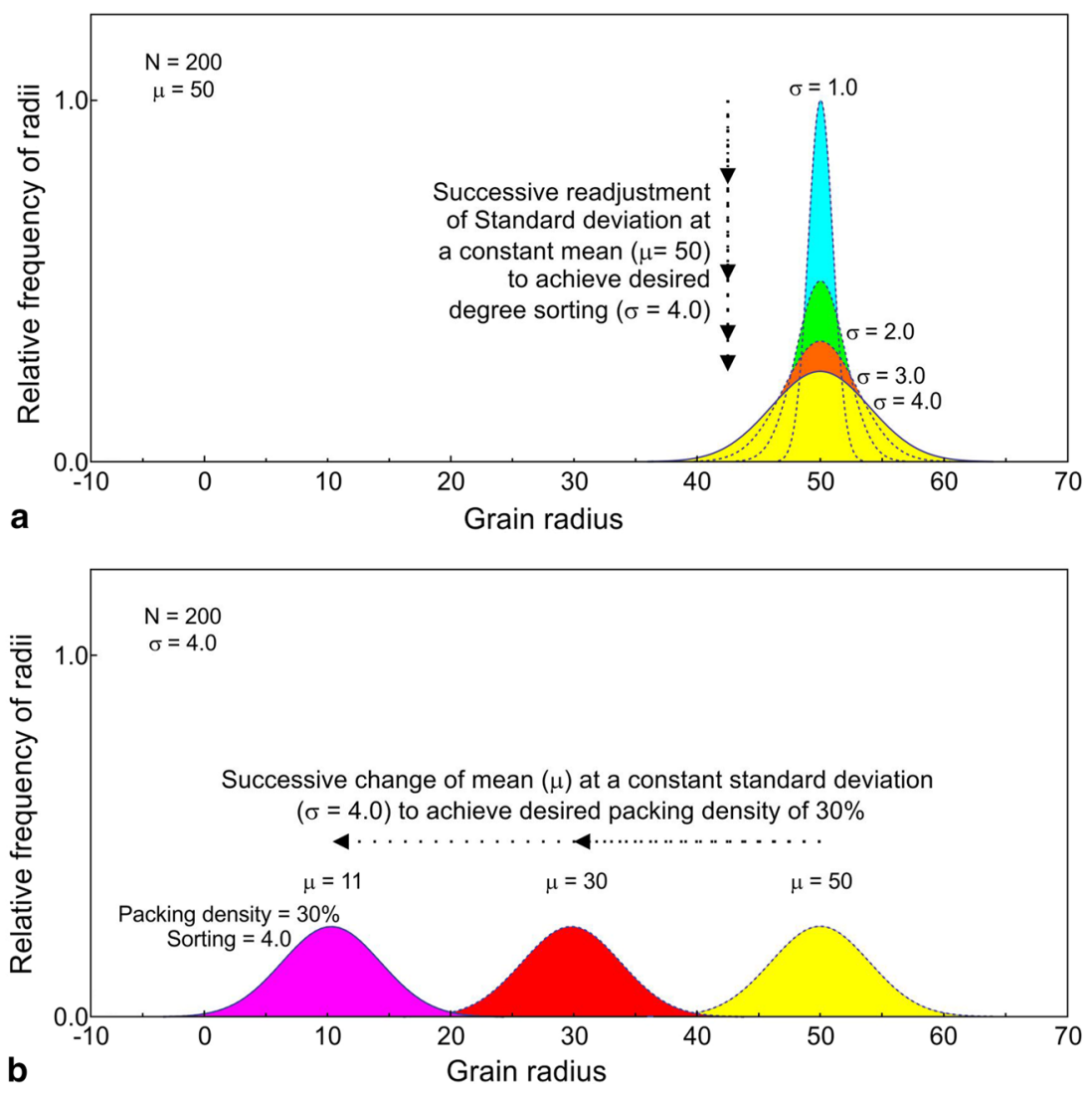

Figure 3. Step-wise procedure for simulation of a sample containing 200 grains of the desired degree of sorting (say, $S=4.0$ ) and packing density (say, $P=30 \%$ ). (a) Different Gaussian curves correspond to different degrees of sorting. The yellow curve with standard deviation, $\sigma=4.0$, is the desired curve. (b) The yellow curve is translated along the X-axis until the desired degree of packing density is achieved. $N$ : number of grains, $\sigma$ : standard deviation and $\mu$ : mean of radii.

Erslev and Ge 1990; Genier and Epard 2007). Although tightly packed aggregates are routinely considered suitable for using the Fry method, the threshold of packing tightness for the Fry method is not yet known. We seek an answer to this question through a series of tests on numerically simulated samples that vary with respect to the packing density and the degree of sorting. Our study assumes the lack of competency contrast between grains and matrix and the homogeneous nature of a constant-volume distortion (Ramsay 1967; Fry 1979; Ramsay and Huber 1983). The rheological contrast due to factors, such as the presence of pore fluids and the grain size variation is also ignored in our simulations. Such an approach is common in the literature on evaluation of assumptions, scope and errors in strain estimation by the Fry method (McNaught 2002; Waldron and Wallace 2007; Lisle 2010; Reddy Vinta and Srivastava 2012; Mulchrone 2013; Kumar et al. 2014). The results of synthetic tests are corroborated by two natural examples.

\section{Methodology}

Graton and Fraser (1935), Kahn (1956), Yerazunis et al. (1962), Griffiths (1967), Blatt et al. (1972), Folk (1974), Vinopal and Coogan (1978) and others have already discussed the issues related to grain size, sorting and packing. In this study, we use the following definition of the packing density, $P$, for simulation of the test samples:

$$
\begin{aligned}
& \text { Packing tightness }(P) \\
& =\frac{\text { area occupied by all the grains }}{\text { total area of the sample }} \times 100 \text {. }
\end{aligned}
$$

Our approach consists of five steps: (i) numerical simulation of 2-D images containing randomly distributed grains of desired packing density \% and sorting, (ii) distortion of the images by known strain ratios, (iii) strain estimation in distorted images by the Fry method, (iv) error estimation, and (v) tests in natural samples.

A brief account of these steps is given below: 

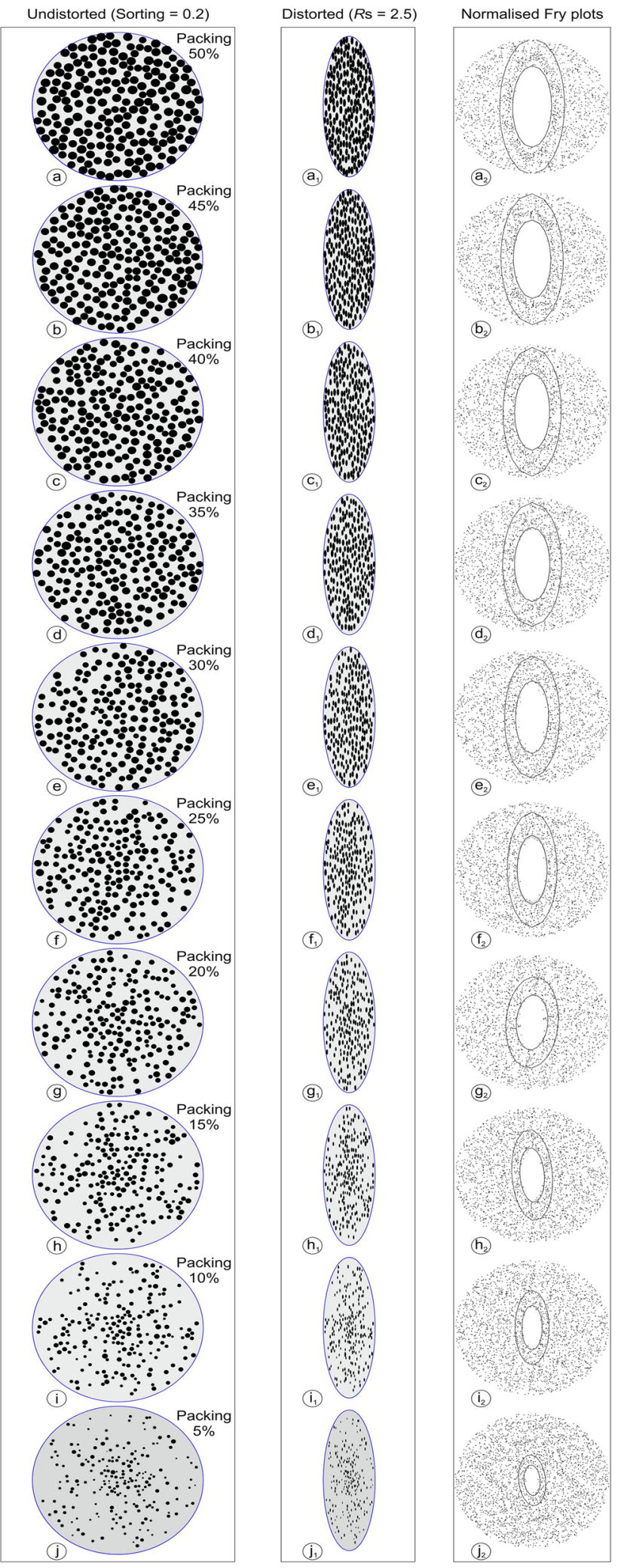

Figure 4. Images of undistorted samples, distorted samples and normalized Fry plots. (a-j) An example of undistorted samples having a constant degree of sorting but varying packing density. $\left(a_{1}-j_{1}\right)$ Distorted samples. $\left(a_{2}-j_{2}\right)$ The best-fit strain ellipses through central vacancy are obtained by the Continuous Function method (Waldron and Wallace 2007). $R s:$ strain ratio.

\subsection{Simulation of the undistorted sample sets}

As poorly sorted samples are not suitable for strain estimation by the Fry method, these are excluded from this study (Waldron and Wallace 2007; McNaught 2002; Reddy Vinta and Srivastava 2012; Mulchrone 2013; Kumar et al. 2014). Our simulations produce 2D-images of the samples such that each sample differs from others with respect to the degree of sorting and the packing tightness. A sample is defined by a circular domain that contains a set of 200 randomly distributed circular grains. For convenience, we define the degree of sorting equal to the standard deviation of radii of all the grains, measured in millimetre (Boggs 2009).

We have developed a MATLAB code, given in the supplementary file, which simulates the 2-D images of undistorted samples. The code requires five user-defined inputs:

(1) radius of the sample, $R$,

(2) number of grains, $N$,

(3) desired degree of sorting, $S$,

(4) desired packing density $\%, P$, and,

(5) maximum run time, $T$ (figure 2).

The value of $R, N, S, P$ and $T$ can be changed as per the requirement. In our simulations, we have fixed $R=20, N=200$ and $T=20$, and varied the degree of sorting and the packing density from 0.2 to 1.0 and 50 to $5 \%$, respectively. We have chosen $R=20$ so that each sample accommodates 200 grains of the desired packing density and sorting. Using these inputs, the code simulates a sample of desired packing density and sorting in the following steps:

(i) It generates the desired number of random grain radii such that each radius is $>0$ (figure $3 \mathrm{a})$.

(ii) It varies the standard deviation of the radii until it equals the desired degree of sorting (figure 3a).

(iii) It achieves the desired degree of packing density $\%$ by varying the mean of the radii, while keeping the standard deviation, obtained in step (ii), fixed (figure 3b). At this stage, we obtain a set of radii that corresponds to the desired degree of sorting and packing density $\%$, respectively.

(iv) Circular grains of different radii, obtained in step (iii) are centred over randomly generated points following the Gaussian distribution in the two-dimensional domain. 

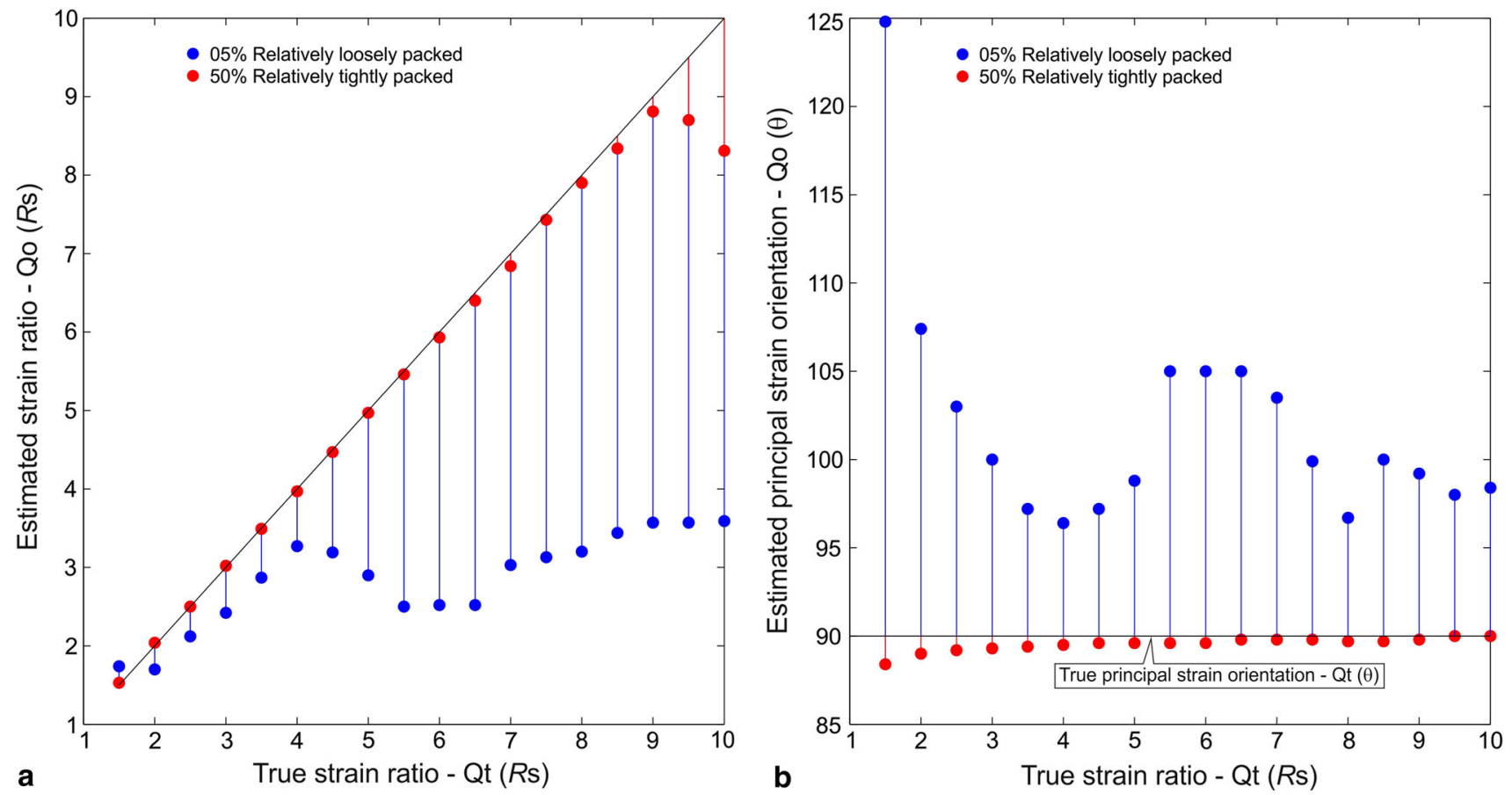

Figure 5. Absolute errors (AE) in two simulated samples having same sorting 0.4 , but different packing density, 5 and $50 \%$. (a) AE in the strain ratio. (b) AE in the principal strain orientation. Irrespective of the true strain ratio, the loosely packed sample, have $5 \%$ packing density, shows larger errors than those in the tightly packed sample of $50 \%$ packing density. $Q_{t}$ and $Q_{o}$ are the true and estimated values, respectively.

(v) A subroutine checks the overlap between grains in the sample obtained in step (iv). If two or more grains overlap, the code regenerates a fresh set of randomly distributed points and centres the circular grains over these points. This process operates iteratively until there are no overlapping grains.

(vi) The event of infinite iteration is checked by a maximum runtime parameter. The terminal harvest is an image that contains nonoverlapping grains of the desired degree of sorting and packing density \% (figure 2).

We have selected five different sets of well-sorted samples that are characterized by the sorting 0.2 , $0.4,0.6,0.8$ and 1.0, respectively. For each of the five selected sample sets, we simulated 10 images of different packing density, 50, 45, 40, 35, 30, 25, 20, 15, 10 and 5\%, respectively (e.g., figure $4 \mathrm{a}-\mathrm{j}$ ). A total of 50 images, having various combinations of the sorting and packing density $\%$, were simulated for the distortion in the next step.

\subsection{Distortion}

We distorted the images by using the following matrix transformation equation for coaxial distortion:

$$
\left[\begin{array}{l}
x^{\prime} \\
y^{\prime}
\end{array}\right]=\left[\begin{array}{ll}
\sqrt{ } R s & 0 \\
0 & 1 / \sqrt{ } R s
\end{array}\right] *\left[\begin{array}{l}
x \\
y
\end{array}\right] .
$$

Here $(x, y)$ and $\left(x^{\prime}, y^{\prime}\right)$ are the co-ordinates of a grain centre before and after distortion respectively. $R \mathrm{~s}$ is the two-dimensional strain ratio.

Each of the 50 images, obtained in the preceding section, was distorted in 18 steps by increasing the strain ratio, $R s$, successively from 1.5 to 10.0 with a constant increment of 0.5 (e.g., figure $4 \mathrm{a}_{1}-\mathrm{j}_{1}$ ). A total of 900 distorted images were obtained for the estimation of errors in samples having various combinations of sorting and packing density $\%$.

\subsection{Error estimation}

Using the Continuous Function method of Waldron and Wallace (2007), we obtained strain estimates from each of the 900 distorted images (e.g., figure $\left.4 a_{2-} j_{2}\right)$. These strain estimates are compared with the known strains, used during numerical distortion of images in section 2.2, for the error analysis.

Errors in the strain estimates can be represented by a variety of statistical measures, each having its merit and limitations (Taylor 1996; Bell 2001). The MAE is a simple linear measure, which has the same unit as that of the observations. Its 

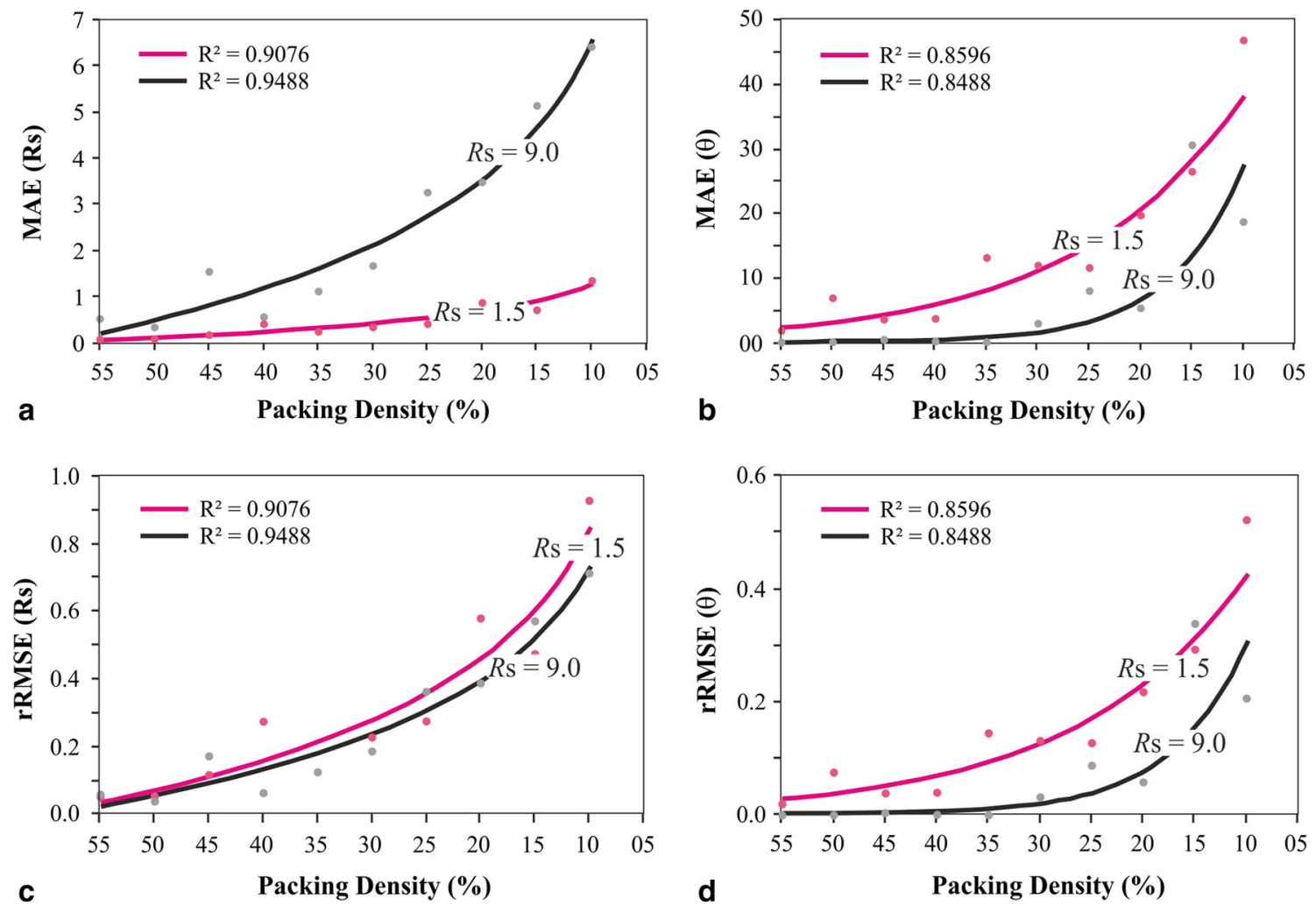

Figure 6. The mean absolute error (MAE) and the relative root mean squared error (rRMSE) in strain ratio $(R s)$ and strain orientation $(\theta)$, measured in degree. The errors increase with decreasing packing density $\%$, irrespective of the level of distortion $(R s) . R^{2}$ : a measure of goodness-of-fit of the regression line.

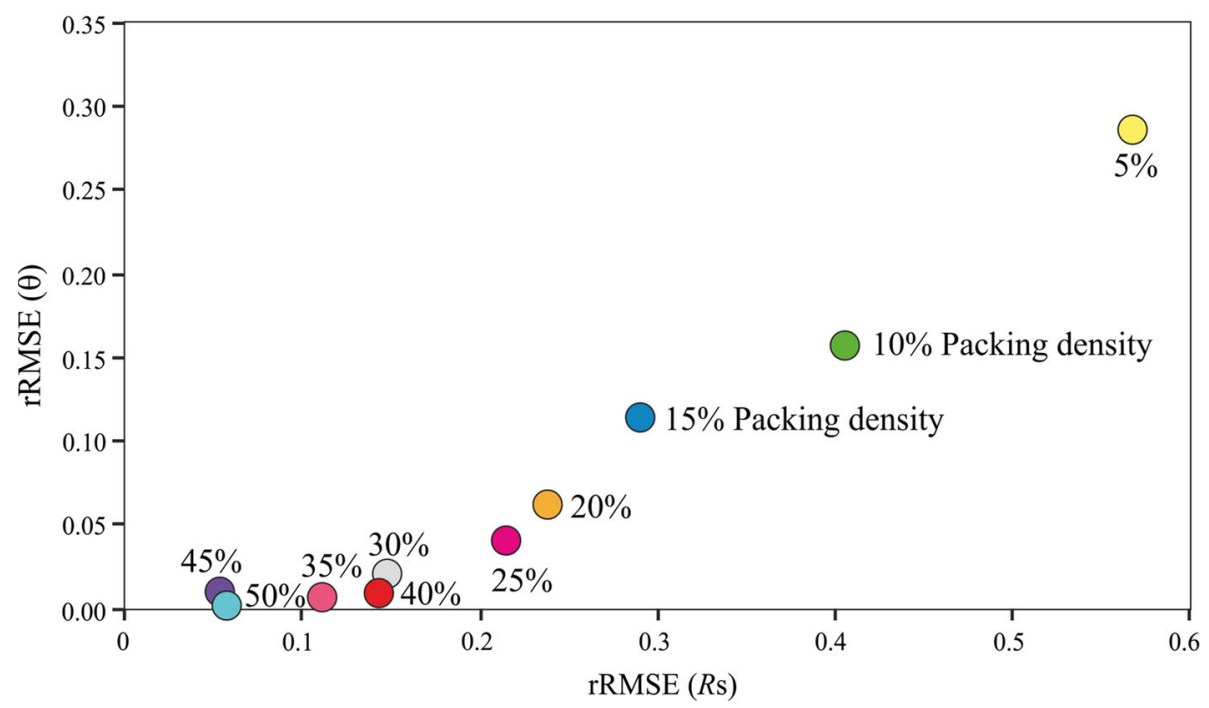

Figure 7. Variation in the rRMSE with respect to the packing density \%. The rRMSE in both, $R s$ and $\theta$, increase sharply in samples having $<30 \%$ packing density.

computation is direct and interpretation more intuitive than the rRMSE (Willmott and Mastuura 2005). The rRMSE scores over the MAE as it accounts for both types of errors: (i) the random error or the variance, and (ii) the systematic error or the bias of the estimator. Another merit of the rRMSE is the tendency to produce several small errors rather than one very large error; two or more small errors of equal magnitude are preferable over a large error. The influence of occasional outliers is larger on the rRMSE because of its quadratic nature and smaller on the MAE due to its linear nature (Willmott et al. 1985; Willmott and Mastuura 2005). The MAE and the rRMSE were 


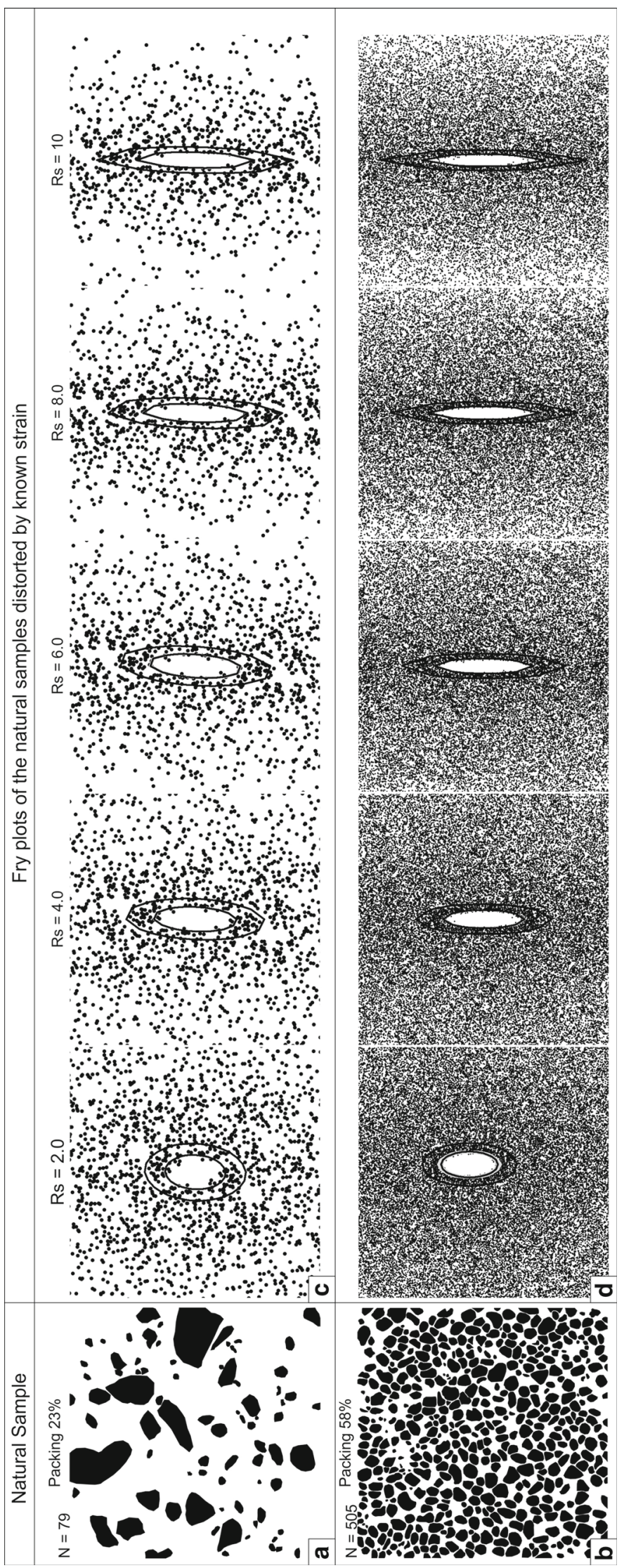


estimated as follows:

The Absolute Error, $\mathrm{AE}=\left|Q_{t}-Q_{o}\right|$,

where $Q_{t}$ and $Q_{o}$ were the true value and the estimated value respectively. $Q$ can either be the strain ratio $(R s)$ or the principal strain orientation $(\theta)$.

$$
\text { The Relative Error, } \mathrm{RE}=\frac{\left|Q_{t}-Q_{o}\right|}{Q_{t}} \text {. }
$$

For a set of $n$ results, the Mean Absolute Error (MAE) and the relative Root Mean Squared Error (rRMSE) are:

$$
\operatorname{MAE}=\frac{\Sigma\left|Q_{t}-Q_{o}\right|}{n},
$$

and

$$
\mathrm{rRMSE}=\sqrt{\frac{\sum\left\{\frac{\left|Q_{t}-Q_{o}\right|}{Q_{t}}\right\}^{2}}{n}} .
$$

Considering the obvious advantages of multiple error measures, we have used AE, MAE and rRMSE in this study. The inferences from all the three measures are found to be consistent.

\section{Results}

\subsection{Synthetic samples}

Figure 5(a-b) shows a typical example of the absolute errors in two such samples that have the same degree of sorting $(S=0.4)$, but different packing density, 5 and $50 \%$ respectively. The absolute errors in the axial ratio $(R s)$ and the orientation $(\theta)$ of the strain ellipse are distinctly larger in the loosely packed sample than those in the tightly packed sample (figure $5 \mathrm{a}-\mathrm{b}$ ). The inference that the AEs become larger with decreasing the packing density $\%$ holds good irrespective of the level of distortion and/or the degree of sorting.

Similar to the AE, the other two error measures, the MAE and the rRMSE also increase with decreasing packing density \% irrespective of the strain ratio and the degree of sorting (figure 6ad). The Cartesian plot between the rRMSE $(R s)$ and the rRMSE $(\theta)$ shows the sensitivity of the Fry method to the packing density \% irrespective of the degree of sorting (figure 7). It is noteworthy
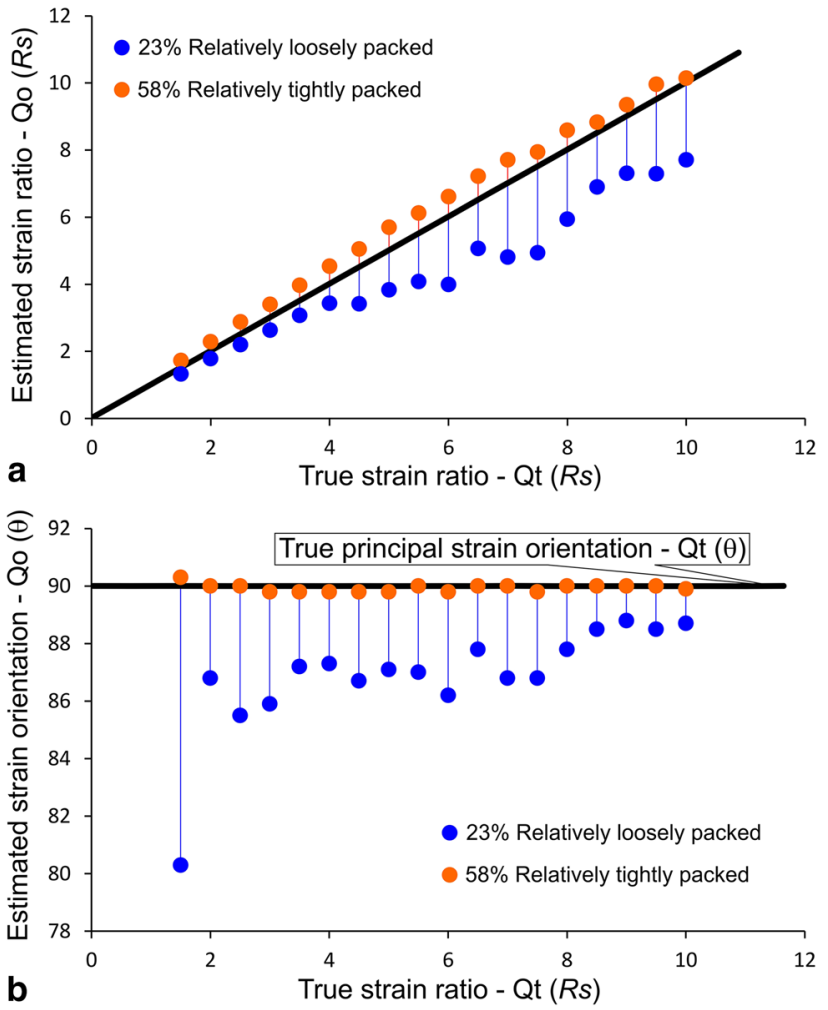

Figure 9. Absolute errors (AE) in two natural samples (figure $8 \mathrm{a}-\mathrm{b}$ ) having different packing density, 23 and $58 \%$. (a) AE in the strain ratio. (b) AE in the principal strain orientation. The loosely packed sample, $23 \%$ packing density, shows larger errors than those in the tightly packed sample of $58 \%$ packing density. $Q_{t}$ and $Q_{o}$ are the true and estimated values, respectively.

that the errors, in particular, increase sharply as the packing density becomes $<30 \%$ (figure 7 ).

\subsection{Natural examples}

In addition to the synthetically simulated samples, we have also tested two natural samples of sandstone. These are: (i) a poorly sorted and loosely packed sandstone from Scotland having $23 \%$ packing density and (ii) a well-sorted and tightly packed Ordovician sandstone having $58 \%$ packing density (figure 8a-b; source: Sand atlas). The images of grain centres in both the samples are distorted by strain ratios ranging from 1.5 to 10.0 , in successive increments of 0.5 . The strain estimates from distorted images are compared with the known strains for error estimation (figure $8 \mathrm{c}-\mathrm{d}$ ).

The results from natural examples substantiate the inferences from synthetic examples. The absolute errors of the axial ratio $(R s)$ and the orientation $(\theta)$ of the strain ellipse are distinctly smaller in the tightly packed sandstone as compared to the loosely packed sandstone (figure $9 \mathrm{a}-\mathrm{b}$ ). 

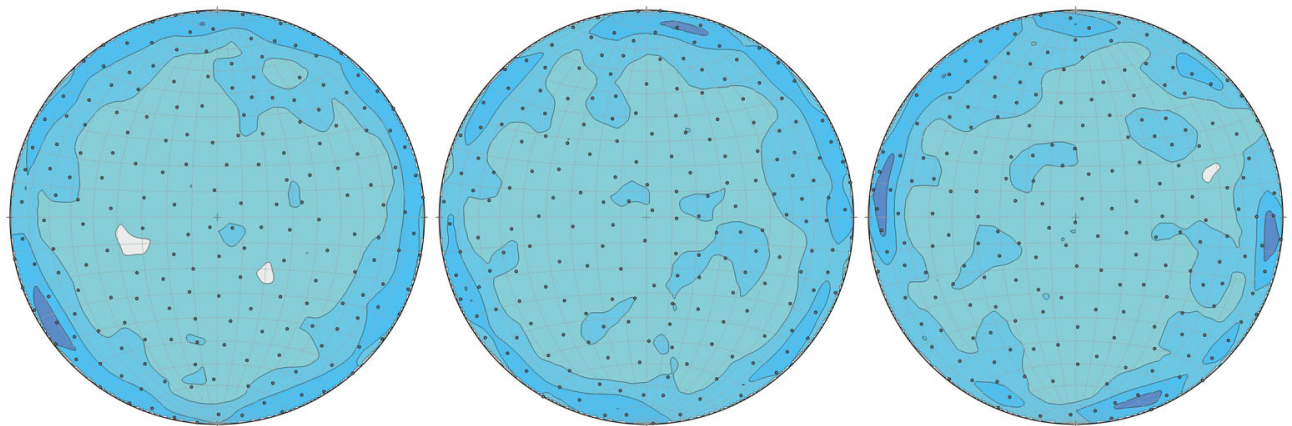

a Packing $50 \%$

b Packing $45 \%$

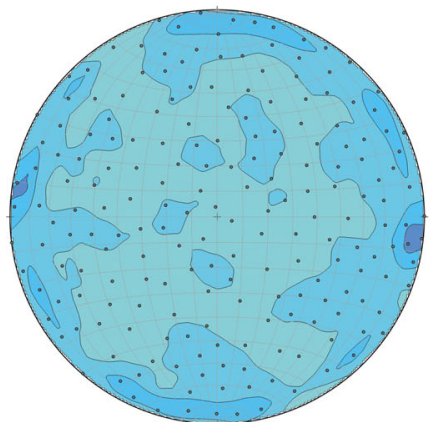

d Packing $35 \%$

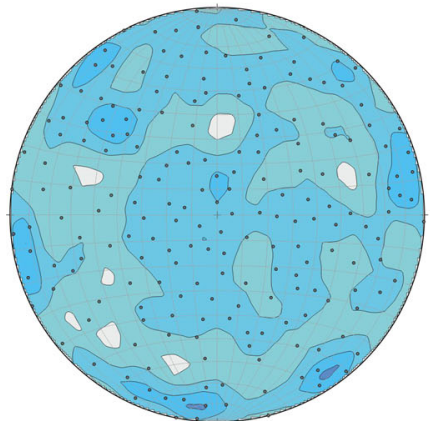

g Packing $20 \%$
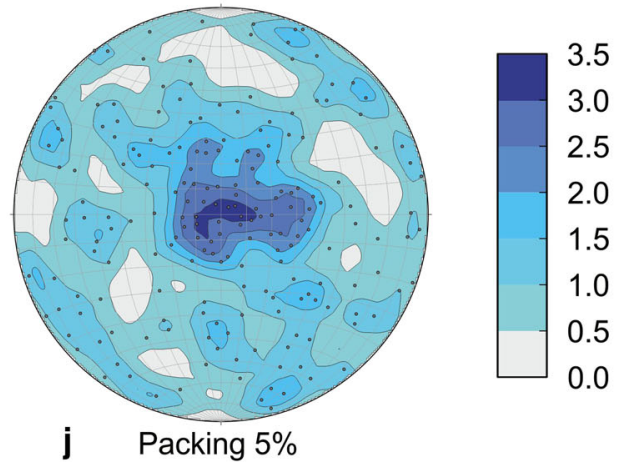

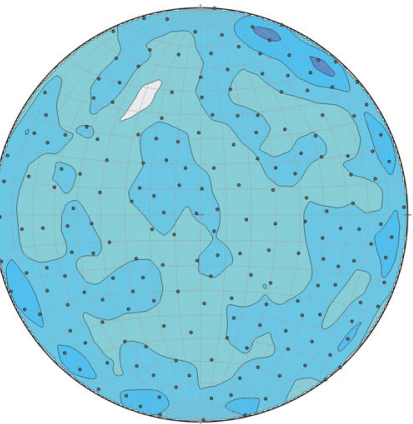

e Packing $30 \%$

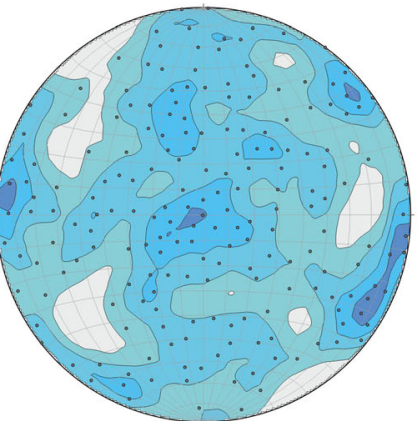

h Packing $15 \%$ c Packing $40 \%$

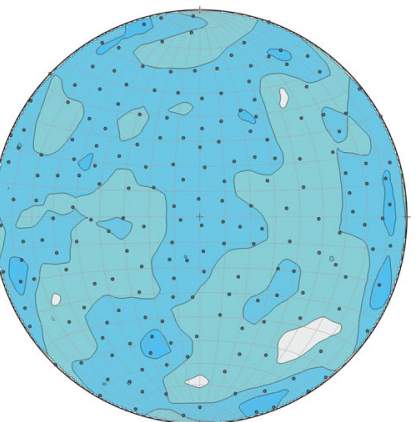

f Packing $25 \%$

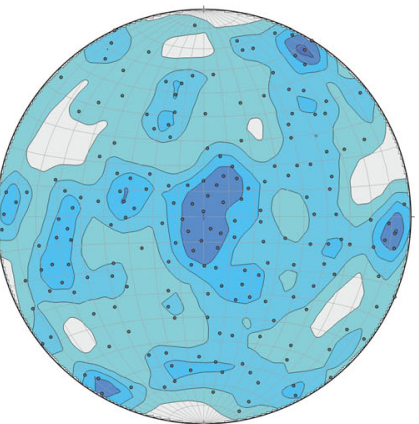

i Packing $10 \%$

Figure 10. Test of clustering in the samples that have a constant sorting, 0.2, but variable packing density. (a-j) Contoured grain centres. Note that distributions become increasingly clustered with decreasing packing density \%. Contours: 0.0-0.5$1.0-1.5-2.0-2.5-3.0-3.5 \%$ per $1 \%$ area. The number of grains in each sample is 200 .

\section{Discussion}

Why do the strain estimates in the loosely packed samples have larger errors? We answer this question by testing the nature of grain centre distribution in the undistorted samples of varied packing density \% (e.g., figure 10).
We use a simple graphical approach that provides a more direct and intuitive interpretation. Alternatively, more sophisticated statistical or analytical methods could also be used for quantitative results (Genier and Epard 2007; Lisle 2010). Equal area contouring is an easy graphical approach for the distinction between clustered and 
anti-clustered point distributions. We have tested the nature of point distributions by contouring the grain centres in the undistorted samples of varied packing density $\%$ and sorting. Figure $10(a-j)$ exemplifies a typical result of such a test on samples shown in figure $4(\mathrm{a}-\mathrm{j})$. Three inferences can be drawn from these results. First, the samples having a higher packing density, $>30 \%$, characteristically lack any point maximum or cluster (figure 10a-c). Second, the point distribution assumes an increasingly clustered nature with the decrease in packing density \% (e.g., figure 10d-i). Third, the point maxima grow and the distribution becomes strongly clustered as the packing density becomes $5 \%$ (figure 10j). Variation in packing density, therefore, affects the degree of clustering in grain centre distribution. In the loosely packed samples, the degree of clustering is stronger and the basic assumption of anti-clustered grain center distribution in the Fry method is violated.

\section{Conclusions}

This study demonstrates that the combination of two parameters, the packing density $\%$ and the degree of sorting, rather than any one of these, need to be considered for testing the suitability of a given sample for strain estimation by the Fry method. Our empirical observations suggest that the packing density must be $>30 \%$, even in wellsorted samples, for strain estimation by the Fry method (figure 7).

\section{Acknowledgements}

This study is supported by J C Bose National Fellowship, SR/S2/JCB/46/2012, of the Department of Science and Technology, Government of India to D C Srivastava. Arun Ojha is supported by the MHRD, Government of India Assistantship. We thank Richard Lisle, an anonymous reviewer, V N Singh and P K Gupta for their constructive reviews of the manuscript and Saibal Gupta for the efficient editorial handling.

\section{References}

Bell S 2001 A beginner's guide to uncertainty in measurements: Guide No 11 (Issue 2); National Physical Laboratory, Middlesex, UK.
Blatt H, Middleton G V and Murray R C 1972 Origin of Sedimentary Rocks; Prentice-Hall, Upper Saddle River, $634 \mathrm{p}$.

Boggs S 2009 Petrology of sedimentary rocks; Cambridge University Press, Cambridge, 600p.

Crespi J M 1986 Some guidelines for the practical application of Fry's method of strain analysis; J. Struct. Geol. 8799 808.

Dacey M F 1964 Two-dimensional random point patterns: A review and interpretation; Paper in Reg. Sci. 13 41-55.

Dunne W M, Onasch C M and Williams R T 1990 The problem of strain-marker centers and the Fry method; J. Struct. Geol. 12 933-938.

Erslev E A 1988 Normalized center-to-center strain analysis of packed aggregates; J. Struct. Geol. 10 201-209.

Erslev E A and Ge H 1990 Least-squares center-to-center and mean object ellipse fabric analysis; J. Struct. Geol. 12 1047-1059.

Folk R L 1974 Petrology of sedimentary rocks; Hemphill Publishing Company, Austin, Texas, 182p.

Fry N 1979 Random point distributions and strain measurement in rocks; Tectonophys. 60 89-105.

Genier F and Epard J-L 2007 The Fry method applied to an augen orthogeneiss: Problems and results; J. Struct. Geol. 29 209-224.

Gonzalez-casado J M, Jimenez-berrocoso A, Garcia-cuevas C and Elorza J 2003 Strain determination using inoceramid shells as strain markers: A comparison of the calcite strain gauge technique and the Fry method; J. Struct. Geol. 25 1773-1778.

Graton L C and Fraser H J 1935 Systematic packing of spheres with particular relation to porosity and permeability; J. Struct. Geol. 43 785-909.

Griffiths J C 1967 Scientific method in analysis of sediments; McGraw-Hill, New York, 508p.

Hanna S S and Fry N 1979 A comparison of methods of strain determination in rocks from southwest Dyfed (Pembrokeshire) and adjacent areas; J. Struct. Geol. 1 155-162.

Kahn J S 1956 The analysis and distribution of the properties of packing in sand-size sediments: 1 . On the measurement of packing in sandstones; J. Geol. 64(4) 385-395.

Kumar R, Srivastava D C and Ojha A K 2014 A comparison of the methods for objective strain estimation from the Fry plots; J. Struct. Geol. 63 76-90.

Lacassin R and Van den driessche J 1983 Finite strain determination of gneiss: Application of Fry's method to porphyroid in the southern Massif Central (France); J. Struct. Geol. 5 245-253.

Lisle R J 1979 Strain analysis using deformed pebbles: The influence of initial pebble shape; Tectonophys. 60 263-277.

Lisle R J 2010 Strain analysis from point fabric patterns: An objective variant of the Fry method; J. Struct. Geol. 32 975-981.

Long S, Mcquarrie N, Tobgay T and Hawthorne J 2011 Quantifying internal strain and deformation temperature in the eastern Himalaya, Bhutan: Implication for the evolution of strain in thrust sheets; J. Struct. Geol. 33 579-608.

McNaught M A 1994 Modifying the normalized Fry method for aggregates of non-elliptical grains; J. Struct. Geol. 6 493-503. 
McNaught M A 2002 Estimating uncertainty in normalized Fry plots using a bootstrap approach; J. Struct. Geol. 24 311-322.

Mulchrone K F 2003 Application of Delaunay triangulation to the nearest neighbour method of strain analysis; $J$. Struct. Geol. 25 689-702.

Mulchrone K F 2013 Fitting the void: Data boundaries, point distributions, and strain analysis; J. Struct. Geol. 46 $22-33$.

Onasch C M 1986 Ability of the Fry method to characterize pressure-solution deformation; Tectonophys. 122 187-193.

Ramsay J G 1967 Folding and fracturing of rocks; McGrawHill, New York, 568p.

Ramsay J G and Huber M I 1983 The Techniques of Modern Structural Geology. Volume 1: Strain analysis; Academic Press, London, 307p.

Ray A and Srivastava D C 2008 Non-linear least square ellipse fitting using the genetic algorithm with applications to strain analysis; J. Struct. Geol. 30 1593-1602.

Reddy Vinta B S S and Srivastava D C 2012 Rapid extraction of central vacancy by image analysis of Fry plots; J. Struct. Geol. 40 44-53.

Sandatlas 2017 http://www.sandatlas.org/sandstone/

Seno S 1992 Finite strain and deformation within the Brianconnais Castelvecchio-Ceriosola nappe of the Ligurian Alps, Italy; J. Struct. Geol. 7 825-838.
Shan Y and Xiao W 2011 A statistical examination of the Fry method of strain analysis; J. Struct. Geol. 33 1000-1009.

Srivastava H B 1995 Two dimensional strain estimation from weakly deformed rocks; An. Tec. 9 3-6.

Taylor J R 1996 An Introduction to Error Analysis: The Study of Uncertainties in Physical Measurements; 2nd edn, University Science Books, California, $448 \mathrm{p}$.

Vinopal R J and Coogan A H 1978 Effect of particle shape on the packing of carbonate sands and gravels; J. Sedim. Res. 48(1) 7-24.

Waldron J W F and Wallace K D 2007 Objective fitting of ellipses in the centre-to-centre (Fry) method of strain analysis; J. Struct. Geol. 29 1430-1444.

Willmott C J, Ackleson S G, Davis R E, Feddma J J, Kink K M, Legates D R, O'donnel J and Rowe C M 1985 Statistics for the evaluation of model performance; J. Geophys. Res. 90 8995-9005.

Willmott C J and Mastuura K 2005 Advantages of the mean absolute error (MAE) over the root mean square error (RMSE) in assessing average model performance; Clim. Res. 30 79-82.

Yerazunis S, Bartlett J W and Nissan A H 1962 Packing of binary mixtures of spheres and irregular particles; Nature 195(4836) 33-35.

Corresponding editor: SAibal Gupta 